\title{
LES CUISINES COLLECTIVES: UNE VOIE VERS LA PROMOTION DE LA SANTÉ MENTALE
}

\author{
SONIA RACINE \\ Moisson Québec \\ et \\ MYREILLE ST-ONGE \\ Institut de réadaptation en déficience physique đu Québec \\ Université Laval
}

\begin{abstract}
RÉSUMÉ
Les cuisines collectives représentent une pratique sociale et communautaire relativement jeune au Québec. Bien connues dans le domaine de l'aide alimentaire aujourd'hui, les cuisines collectives dépassent largement le soutien alimentaire par leurs effets sur les conditions de vie et le développement personnel des personnes qui $s^{t} y$ engagent. Cette recherche qualitative tente d'éclairer les différentes retombées des cuisines collectives sur les participantes et les participants dans une optique de promotion de la santé mentale, et de dégager les facteurs en fonction desquels celles-ci émergent. Trois types de facteurs sont relevés, c'est-à-dire ceux reliés à la dynamique interne de chacun des groupes de cuisine collective, les facteurs personnels et ceux reliés à l'utilisation d'autres services et à l'engagement social des participantes et des participants.
\end{abstract}

\section{INTRODUCTION}

Depuis plusieurs années, le Québec et le Canada traversent des mutations profondes quant à leurs structures sociales et économiques. Les conditions du marché du travail et les politiques de plus en plus restrictives en matière de protection sociale sont quelques éléments explicatifs de l'appauvrissement d'une grande partic de la population. En 1996, au Canada, $17,6 \%$ de la population était considérée pauvre, tandis que ce pourcentage s'élevait à $21,2 \%$ au Québec (Conseil national du bien-être social, 1998). L'image de la pauvreté se transforme; on assiste entre autres à une féminisation de la pauvreté, plus particulièrement pour les jeunes

Nous désirons exprimer nos plus sincères remerciements à Elaine Côté, Céline Morrow et Esther Tremblay qui ont participé à l'ensemble de ce projet de recherche et collabort́ a la rédaction du rapport final, ainsi qu'aux trois évahuateurs et évaluatrices anonymes pour leurs judicieux commentaires. Cetre recherche a été financée par la Régie régionale de la Santé et des Services socjaux de la région de Québec. Elle a remporté le Prix d'excellenec provincial dans la catégorie -Organisme communatuaire. du programme de subventions en santé publique du ministère de la Santé et des Services sociaux du Quéthec. Les demandes de tirtes à part doivent ètre adresséces à Sonia Racine, Moisson Québee, 12-1375, boul. Charest ouest, Québec (QC), G1N 2 E7. 
familles monoparentales. Son intensité et sa persistance ont également tendance à se modifier. En effet, il y a une augmentation du degré de précarité, c'est-à-dire l'écart entre le revenu réel et le seuil de pauvreté, et la situation de pauvreté persiste sur une période de plus en plus longue. Dans une situation de pauvreté, les facteurs de risque pouvant porter atteinte à la santé mentale sont nombreux: logement insalubre, absence de pouvoir, désespérance, stress, isolement, honte, etc. Non seulement la pauvreté augmenterait les risques de problèmes de santé mentale, mais elle aggraverait les conséquences de ces problèmes (Robichaud, Guay, Colin, \& Pothier, 1994). Rappelons également que les conclusions des enquêtes Santé Québec de 1987 et de 1992-93 ont confirmé l'existence du lien entre la pauvreté et une plus grande đétresse psychologique (Santé Québec, 1989. 1994).

Pour pallier à cette pauvreté grandissante, de nombreux organismes communautaires sont apparus. Afin de résorber les problèmes de malnutrition et de faim dans notre société, beaucoup d'entre eux ont développé des projets alimentaires variés, dont les cuisines collectives. En avril 1999, dans l'agglomération de la ville de Québec, quelque 150 organismes accrédités à Moisson Québec offrent divers services de dépannage alimentaire (colis de nourriture, bons d'épicerie, etc.) ou de repas (soupes populaires, collations dans les écoles, etc.), soutenant ainsi plus de 24000 personnes par mois. Les quantités de nourriture distribuées indiquent bien à quel point les besoins sont grandissants: en 1988, 500266 kilos de nourriture ont été distribués par Moisson Québec, alors qu'elle en distribuait plus de 2100000 kilos en 1999 (Moisson Québec, 1999). Depuis leur émergence dans la région de Québec en 1990, les cuisines collectives ont pris une ampleur insoupçonnée. Selon l'Association des cuisines collectives et créatives de la région de Québec (1999), il en existerait, en avril 1999, plus de 120 réparties dans une soixantaine d'organismes, plusieurs groupes de cuisine collective pouvant être coordonnés par le même organisme. Étant donné la grande mouvance de ce milieu, il est difficile de tenir des données exactes à ce sujet. Des groupes de cuisine collective émergent à tous moments, alors que d'autres cessent leurs activités pour une courte période ou de façon définitive.

C'est dans ce contexte de croissance des besoins et des multiples formes d'aide pour y répondre que cette étude a été réalisée. Elle visait à identifier les motivations et les attentes des participantes et des participants face aux cuisines collectives, à connaitre leur perception des divers effets de celles-ci sur leur situation personnelle et leur santé mentale, et surtout à comprendre les processus dynamiques en oeuvre dans l'émergence de ces effets. Lorsque l'on traite ici de la santé mentale, on renvoie à la notion de détresse psychologique qui est définie comme un état subjectif déplaisant et non à ce que l'on qualifie de troubles mentaux (Mirowski \& Ross, 1989).

\section{La pratique des cuisines collectives}

En dépit des diverses réalités qui traversent cette pratique sociale, on définit gênéralement une cuisine collective comme étant un groupe de quatre à cinq personnes mettant en commun leur temps, énergie et compétences pour préparer des repas sains et équilibrés à moindre coût (Montminy, 1991; Regroupement des cuisines collectives du Québec, 1996). Elles existent depuis 1986 au Québec et 
depuis plus de 20 ans en Amérique latine. Le principe des cuisines collectives tirerait son origine de l'expérience des femmes péruviennes dans les bidonvilles qui constituent désormais une force politique importante (Fréchette \& Fayreau, 1997). Il existe une multiplicité d'angles et de méthodologies par lesquels il est possible d'examiner les cuisines collectives. À ce jour, nous avons répertorié sept études au Canada traitant spécifiquement de ce sujet dans des perspectives fort différentes (Deslauriers, 1998; Fournier, Provost, \& Goudreault, 1998; Fréchette \& Favreau, 1997: Kalina, 1993; London Good Food Project, 1993; Noraz, 1996; Tremblay. 1995). Nous tenterons done de faire ressortir les faits saillants nous permettant de mieux saisir cette pratique en pleine effervescence sur le plan de l'amélioration des conditions de vie et de santé mentale des personnes qui y participent. Bien que plusieurs des recherches répertorices aient largement contribué à développer d'autres aspects des cuisines collectives, notamment sur le plan de leur impact communautaire, nous ne les évoquerons pas dans cet article afin de se centrer sur T'angle de notre recherche.

Sur le plan descriptif, notons que les cuisines collectives rejoignent principalement, mais non exclusivement, des femmes entre 30 et 45 ans, chefs de famille monoparentale, sans emploi et provenant de milieux socio-économiquement défavorisés. Les cuisines collectives accueillent également d'autres types de personnes: familles traditionnelles, familles monoparentales dirigées par un homme, adolescents et adolescentes, personnes ayant un problème de santé mentale, personnes âgées, etc. Bien que présentant des caractéristiques différentes, les participantes et les participants aux cuisines collectives ont un point en commun, celui d'être plongés dans une précarité sociale et économique (Tremblay, 1995). Certains groupes visent des clientèles particulières même si ce n'est pas le cas pour la majorité. Quant aux objectifs, on remarque qu'ils gravitent généralement autour de la lutte contre la faim, l'insécurité alimentaire et la pauvreté, du bris de l'isolement social, de la dimension éducative (nutrition, consommation, etc.) et de l'entraide (Deslauriers, 1998; Fournier et al. 1998; Fréchette \& Favreau, 1997: Tremblay, 1995).

Très diversifiées à tous points de vue, les cuisines collectives ont également des orientations générales qui leur sont propres. La majorité sont davantage centrées sur la nutrition, d'autres sur l'entraide ou encore sur le développement communautaire (Fréchette \& Favreau, 1997). Les objectifs et les orientations qu'elles adoptent influencent d'ailleurs fortement à la fois leur fonctionnement, leur dynamique et les effets qui en découlent sur les personnes qui y participent (Fournier et al., 1998; Fréchette \& Favreau, 1997). Tremblay (1995) a par ailleurs montré que dans 13 des 33 groupes étudiés, les participantes et les participants n'étaient pas engagés dans le processus décisionnel menant à la répartition des taches. L'analyse approfondie de ces variations et du fonctionnement dynamique des cuisines ne constituait pas l'objet de cette première étude dans la région de Québec, mais les résultats obtenus laissent croire que le degré de participation et d'engagement puisse varier considérablement d'une cuisine à l'autre et que les effets sur la qualité de vie des usagères et des usagers puissent en ce sens en être tributaires.

Les cuisines collectives diffèrent quant au nombre de participantes et de participants, à la nature et au nombre de mets cuisinés, à la fréquence des 
rencontres, à la provenance et au róle des responsables et animatrices (toutes les personnes qui étaient responsables ou animaient des groupes de cuisine collective au moment de la recherche étant des femmes, nous utiliserons donc le genre féminin pour les décrire), à leur organisme d'appartenance, à leur financement, etc. Parmi les caractéristiques communes, on note l'engagement humain et financier des personnes qui en font partie bien qu'à des degrés fort variables (a l'opposé d'une pratique de service où ces personnes ne sont que des consommateurs et consommatrices), un haut taux de roulement, des difficultés de financement ainsi que des retombées avantageuses pour les participantes et les participants sous plusieurs aspects (Deslauriers, 1998; Fournier et al. 1998; Fréchette \& Favreau, 1997; Tremblay, 1995).

Toutes les recherches relèvent une augmentation ou une consolidation de la confiance en soi et de l'estime personnelle. Il en est de même sur le plan de l'acquisition de connaissances et d'habiletés personnelles (principalement culinaires et nutritionnelles), de la création de liens sociaux et affectifs (augmentation du soutien social), de l'augmentation des habiletés sociales, de la quantité, variété et qualité de l'alimentation, de la réalisation d'économies substantielles qui aiderait à la diminution du stress, ainsi que de la connaissance des diverses ressources institutionnelles ou communautaires. Fournier et al. (1998) et Fréchette et Favreau (1997) mentionnent également des retombées sur le plan communautaire, tels qu'un engagement accru des personnes dans leur milieu, une prise en charge collective des situations de vie et une diminution des préjugés; alors que Deslauriers (1998) et Noraz (1996) soulignent le cheminement des participantes et des participants vers l'élaboration de projets personnels (retour aux études, bénévolat, recherche d'emploi, etc.). Ainsi, dans un sens large, les cuisines collectives contribueraient à favoriser l'insertion sociale des participantes et des participants (Deslauriers, 1998; Fournier et al., 1998; Fréchette \& Favreau, 1997; Noraz, 1996).

Selon les auteures et les auteurs des recherches répertoriées, les retombées de la participation ne sont pas uniquement attribuables aux cuisines collectives comme telles, mais également à de nombreux autres facteurs qui ne relèvent pas expressément de leur dynamique interne. La participation à d'autres activités communautaires, l'engagement dans d'autres projets de même que des changements sur le plan personnel influencent le cheminement des personnes à travers leur participation aux cuisines collectives. Au chapitre des éléments organisationnels et de dynamique interne pouvant expliquer l'importance des retombées des cuisines collectives sur les participantes et participants et leur réussite sociale, le fort sentiment d'appartenance à un groupe, la qualité des relations vécues à l'intérieur de celui-ci, la convivialité, le processus participatif favorisant de multiples échanges, la souplesse (peu de formalisation) et le plaisir d'être ensemble semblent des ćléments clés (Fournier et al., 1998; Fréchette \& Favreau, 1997; Kalina, 1993; London Good Food Project, 1993; Noraz, 1996).

Avec des perspectives variées, ces différentes études nous permettent de mieux connaitre ce que sont les cuisines collectives dans un contexte social, économique, culturel et politique comme le nôtre. Il faut toutefois saisir l'importance du développement inégal et différencié de cette pratique avant tout communautaire, dans les régions du Québec et à travers le Canada, et son influence sur les résultats de recherche. 
Un double cadre conceptuel:

la promotion de la santé mentale et l'aide mutuelle

L'angle d'étude avec lequel nous abordons les cuisines collectives présente deux aspects; d'abord le potentiel de celles-ci à promouvoir la santé mentale (au sens large du bien-être psychologique) dans un contexte de pauvreté risquant de lui porter atteinte; ensuite, une dynamique propre aux cuisines collectives permettant de saisir les processus en auvre.

De façon générale, on peut dire qu'un des grands principes devant guider l'action des groupes offrant des services d'aide alimentaire constitue la création de conditions favorables à la prise de pouvoir des participantes et participants et de mise à profit de leurs capacités afin qu'ils s'assurent leur propre sécurité alimentaire et reprennent du contrôle sur leur vie (Beeman, Panet-Raymond, \& Rouffignat, 1997). Cette idée se rapproche éminemment de la philosophie des cuisines

\section{TABLEAU 1}

Les déterminants de la santé mentale

\section{Valeurs sociales et culturelles}

- Degré de tolérance vis-à-vis des differences

- Compétition vs coopération

- Automonie vs dépendance

- Individualisme vs collectivisme
Environnement politique

- Présence ou absence de politiques sociales

- Présence ou absence de concertation intersectorielle au niveau gouvernemental, en lien avec la santé mentale
Facteurs biogénétiques

- Vieillissement

- Déficience physique de naissance

\section{Conditions économiques}

- Niveau et sécurité du revenu

- Changement économique rapide (croissance ou récession)

- Emploi et non-emploi

- Infrastructures économiques

\section{Ressources psychologiques personnelles}

- Habiletés de communication, de verbalisation des émotions, de résolution de problèmes

- Estime de soi

- Compétences sociales

\section{Habitudes de vie}

- Gestion de la consommation d'alcool

- Activités physiques

\section{Quantité et qualité des services}

- Disponibilité des services

- Répartition des ressources en fonction des besoins: compatibilité, congruence, continuité

- Horaires compatibles

- Ressources aux familles: garderies, services de gardiennage, services de répit

\section{Relations de soutien}

- Qualité des róles occupés dans divers milieux

- Qualité des relations interpersonnelles

- Accès à des sources de soutien variées

\section{Environnement physique}

- Conditions de logement: salubrité, bruit, coûts

- Présence ou absence de toxiques

- Accès aux espaces verts

Source: Ministère de la Santé et des Services sociaux (1990). 
collectives et de celle de la promotion de la santé mentale telle que définie par Blanchet, Laurendeau, Paul et Saucier (1993, p. 15):

La promotion vise l'accroissement du bien-être personnel et collectif en développant les facteurs de robustesse et les conditions favorables à la santé mentale. Son action porte sur les déterminants de la santé plutôt que sur les facteurs de risque, et vise la population générale ou des sous-groupes particuliers.

Les déterminants de la santé mentale sont nombreux et interagissent dans toutes les sphères d'activités de la vie d'un individu. Nous présentons au tableau 1 l'ensemble des déterminants de la santé mentale tels que définis par le ministère de la Santé et des Services sociaux du Québec (1990) afin d'être en mesure de faire un parallèle entre les effets perçus de la participation aux cuisines collectives chez les répondantes et les répondants et leur potentiel de promotion de la santé mentale.

C'est à partir du concept d'aide mutuelle comme processus par lequel les membres d'un groupe donné en viennent à partager et à échanger de différentes façons dans le but de s'offrir mutuellement du soutien que nous analyserons les processus présents dans les cuisines collectives. Basé sur le principe de l'entraide entre les participantes et participants, le concept apparaît donc tout à fait pertinent pour étudier les processus dynamiques en oeuvre. Nous reproduisons au tableau 2 la synthèse de Renaud (1993) à ce sujet.

L'objet de notre étude consistait donc à pousser davantage les connaissances des effets des cuisines collectives sur les conditions de vie des participantes et des participants, particulièrement au chapitre de la promotion de la santé mentale, et surtout à établir le lien qui existe entre ces différentes retombées et les processus dynamiques en cuvre selon la perception des personnes qui y participent. II s'agissait ainsi d'explorer l'ensemble des effets perçus et d'y associer les éléments du fonctionnement ou de la structure des cuisines collectives identifiés comme motifs de ces effets. Tout au cours de cette recherche, nous avons établi un partenariat solide avec les milieux communautaire et institutionnel, ce qui favorise des retombées concrètes pour l'action immédiate. En raison du contexte d'appauvrissement, de la multiplication des services d'aide alimentaire et des interrogations persistantes sur les modes d'intervention en santé mentale, cette recherche d'exploration sur les cuisines collectives, comme activité potentielle de promotion de la santé mentale, nous semble essentielle. Il est primordial que les intervenantes et les intervenants préoccupés par la problématique de la pauvreté puissent connaître et comprendre les mécanismes de promotion de la santé mentale émanant de la participation aux cuisines collectives afin de les renforcer et d'être en mesure de les adapter à d'autres types d'interventions.

\section{MÉTHODOLOGIE}

Pour répondre à l'objet de recherche, nous avons opté pour un devis qualitatif de type exploratoire. La technique principale de collecte des données retenue est l'entrevue face à face de type semi-structuré (questions ouvertes). Par ailleurs, nous avons eu recours à des entretiens téléphoniques avec quelques responsables de cuisine collective et à deux observations participantes lors de journées de cuisine afin de constituer l'échantillon et de nous aider à développer les outils de recherche (guide d'entrevue et grille d'analyse des données). 


\section{TABLEAU 2}

La dynamique de Paide mutuelle

\begin{tabular}{|c|c|}
\hline Processus & Caractéristiques \\
\hline Partage de données & $\begin{array}{l}\text { - Partager des expériences vécues } \\
\text { - Exprimer ses idées } \\
\text { - Partager ses cornaissances } \\
\text { - Exprimer ses croyances } \\
\text { - Offrir son point de vue }\end{array}$ \\
\hline Processus d'échange & $\begin{array}{l}\text { - Exprimer ses différences } \\
\text { - Apporter une opinion ou une perception } \\
\text { differente } \\
\text { - Confronter ses idées à celles des autres } \\
\text { - Dégager une synthèse } \\
\text { - En arriver à une nouvelle compréhension }\end{array}$ \\
\hline Discussion de tabous & $\begin{array}{l}\text { - Parler de sujets socialement cinterdits: } \\
\text { - Révéler aux autres des aspects de soi-méme qu'on } \\
\text { n'aime pas ou des comportements qu'on croit } \\
\text { inappropriés }\end{array}$ \\
\hline
\end{tabular}

Tous dans le même bateau

- Découvrir que tous partagent les mémes sentiments, problèmes, besoins

\begin{tabular}{|c|c|}
\hline Soutien mutuel & $\begin{array}{l}\text { - Accepter les autres } \\
\text { - Comprendre sans juger } \\
\text { - Offrir de l'empathie } \\
\text { - Réconforter }\end{array}$ \\
\hline Demandes et attentes mutuelles & $\begin{array}{l}\text { - Écouter les autres } \\
\text { - Confronter les idées arrêtées, les préjugés, les } \\
\text { blocages et les négations empêchant la } \\
\text { résolution de problèmes communs }\end{array}$ \\
\hline Résolution de problèmes personnels & $\begin{array}{l}\text { - Offrir un point de vue differrent et suggérer des } \\
\text { solutions de remplacement }\end{array}$ \\
\hline Répétition & $\begin{array}{l}\text { - Risquer de nouvelles habiletés dans un groupe } \\
\text { - Pratiquer ces habiletés dans sa vie quotidienne } \\
\text { - Vérifier la mise en pratique des habiletés }\end{array}$ \\
\hline & - Agir collectivement \\
\hline
\end{tabular}

Source: Renaud (1993), pp. 110-116. 


\section{Le processus d'échantillonnage et les critères d'inclusion}

Pour construire notre échantillon, nous avons misé sur la diversification, tant au plan du type de cuisine (sachant que les orientations et les autres caractéristiques des groupes peuvent en influencer les retombées) que du type de personnes qui y participent. Les groupes de cuisine collective ont d'abord été sélectionnés selon la diversité de leur fonctionnement et de leur secteur géographique d'activité. Il est à noter que seules les cuisines collectives sur le territoire de la Régie régionale de la Santé et des Services sociaux de Québec ont été retenues pour cette étude. Celles des comtés de Portneuf et de Charlevoix n'ont pas été contactées, principalement pour des raisons financières, et aussi à cause de la faible quantité de cuisines collectives dans ces comtés.

Une fois les groupes sélectionnés, les participantes et les participants devaient répondre à certains critères: être engagés depuis au moins six séances dans des activités de cuisine collective au moment de l'entrevue (cette fréquence a été fixée à partir des commentaires de responsables de cuisines collectives et d'intervenantes engagées dans leur développement depuis leurs débuts dans la région de Québec) et être âgés entre 18 et 60 ans. Dans le but de bien refléter le profil des participantes et des participants des cuisines collectives tel que défini par Tremblay (1995), nous avons opté pour une technique d'échantillonnage empirique, soit l'échantillonnage par quotas ou stratifié (Beaud, 1990). Nous présentons au tableau 3 les critères de diversification qui ont été appliqués.

\section{TABLEAU 3}

Ratios appliqués dans la constitution de P'échantillon d'après Tremblay (1995)

\begin{tabular}{llc}
\hline Critères & Catégories & Pourcentage \\
\hline Sexe & - Femmes & $83 \%$ \\
& - Hommes & $17 \%$ \\
\hline Àge & $\cdot 18-30$ ans & $22 \%$ \\
& $-31-45$ ans & $66 \%$ \\
& $-46-60$ ans & $12 \%$ \\
\hline Statut familial & - Monoparentalité & $66 \%$ \\
& - Biparentalité & $19 \%$ \\
& - Sans conjoint ni enfant & $16 \%$ \\
& - Autres & $6 \%$ \\
\hline
\end{tabular}

Source: Racinc, Côté, Morrow, St-Onge, \& Tremblay (1997).

Deux étapes principales ont été requises afin de constituer l'échantillon de la recherche. En premier lieu, une lettre explicative du projet a été adressée à toutes les responsables de cuisine collective de la rive-nord de la région de Québec. Par la suite, un contact téléphonique a été effectué avec chacune des responsables afin de connaitre son intention de participer à la recherche. La deuxième étape a consisté à recruter les participantes et les participants à l'intéricur des cuisines collec- 
tives sélectionnées. Environ la moitié des participants et des participantes ont été rejoints de cette façon. Ensuite, pour que notre échantillon corresponde à la stratification prédéterminée, nous avons sollicité des personnes selon les ratios établis. Cette méthode s'est avérée efficace afin d'atteindre des clientèles plus difficiles à rejoindre (hommes, jeunes, etc.). La constitution de l'échantillon stratifié a été effectuée en même temps que la réalisation des entrevues, ce qui a permis d'arrêter les démarches lorsque la saturation de contenu a été atteinte (Mayer \& Ouellet, 1991).

\section{Les thèmes retenus}

Quatre blocs de questions ont été développés touchant les sujets suivants: (a) le fonctionnement de la cuisine collective, principalement en rapport avec les processus décisionnels, la répartition des tâches, l'ambiance et les relations interpersonnelles; (b) les débuts de participation à la cuisine, notamment sur le plan de la mise en contact avec la cuisine, des motivations à y participer, des craintes et apprébensions qui y étaient reliées, de la situation préalable de la personne (santé mentale, événements perturbateurs, etc.) ainsí que des attentes face à cette activité; (c) les effets perçus et leur processus dynamique, principalement en lien avec l'état de santé mentale préalable, les facteurs qui, dans la cuisine collective, ont contribué à produire ces effets et l'utilisation d'autres services ou événements ayant pu les influencer; et (d) l'appréciation générale de la cuisine collective, en insistant sur ce que les participantes et les participants ont aimé le plus dans leur expérience, ce qu'ils ont aimé le moins et leurs suggestions pour améliorer cette activité. Ce guide d'entrevue a été prétesté auprès de 2 participantes. À la suite de ces deux entrevues, nous n'avons procédé qu’à des modifications mineures du guide. Ces entrevues ont donc été conservées dans l'échantillon final puisque les thèmes sont demeurés inchangés.

\section{Les répondantes et les répondants}

Vingt-cinq entrevues d'une durée moyenne de 90 minutes ont été réalisées principalement au domicile des participantes et des participants. Les objectifs de la recherche ainsi que les thèmes à discuter leur étaient expliqués et ils étaient invités, par la suite, à lire et à signer un formulaire de consentement leur assurant la confidentialité des données recueillies. Les 25 personnes répondantes dont 20 femmes $(80 \%)$ et 5 hommes $(20 \%)$ étaient âgées entre 20 et 60 ans. Ces personnes provenaient de 13 organismes différents, situés dans divers secteurs. Il convient de mentionner que des 25 répondantes et répondants, 4 étaient responsables de leur groupe au moment de l'entrevue, ce qui leur attribue une particularité importante sur laquelle nous reviendrons. Le tableau 4 présente un profil détaillé de l'échantillon. Ce tableau nous permet de constater la diversité des participantes et participants sur le plan sociodémographique et il est possible d'y constater que les ratios déterminés ont été respectés. Les ratios délimités par la recherche de Tremblay (1995) ont été sensiblement dépassés pour certains critères. Cependant, la diversification de plus en plus grandissante des personnes engagées dans les cuisines collectives (de plus en plus d'hommes, de jeunes et de familles biparentales) nous pousse à croire que l'échantillon est tout à fait représentatif de la situation qui prévaut dans la région de Québec. 


\section{TABLEAU 4}

Profíl des répondantes et des répondants $(\mathrm{N}=25)$

\begin{tabular}{lcclccc}
\hline Caractéristiques & N & $\%$ & Caractéristiques & N & $\%$ \\
\hline Groupe d'âge & & & Statut familial & & \\
$18-30$ ans & 6 & 24 & Familles monoparentales & 12 & 48 \\
$31-45$ ans & 15 & 60 & Familles biparentales & 7 & 28 \\
$46-60$ ans & 4 & 16 & Personnes seules & 3 & 12 \\
& & & Couples & 3 & 12
\end{tabular}

Occupation principale

Éducation des enfants

Emploi rémunéré

Emploi non rémunéré

En recherche d'emploi

Aux études

Sans emploi

\section{Degré de scolarité}

Secondaire non terminé

Secondaire terminé

Collégial

Universitaire

$\begin{array}{cc}10 & 40 \\ 4 & 16 \\ 3 & 12 \\ 3 & 12 \\ 3 & 12 \\ 2 & 8\end{array}$

\section{Source principale de revenu}

Sécurité du tevenu

Travail rémunéré (salaire)

Assurance-emploí

Pension

$\begin{array}{cc}16 & 64 \\ 7 & 28 \\ 1 & 4 \\ 1 & 4\end{array}$

\section{Durée de la participation*}

Moins d' 1 an

De 1 à 2 ans

De 2 à 3 ans

Plus de 3 ans

$\begin{array}{cc}7 & 28 \\ 10 & 40 \\ 4 & 16 \\ 4 & 16\end{array}$

Fréquence de la participation

$\begin{array}{lcc}1 \text { fois / mois } & 19 & 76 \\ 2 \text { fois / mois } & 1 & 4 \\ 3 \text { fois / mois } & 2 & 8 \\ 4 \text { fois / mois } & 3 & 12\end{array}$

- Notons que l'étendue de la participation varie entre 3 mois et 5 ans et 8 mois et que la moyenne se situe i) 1 an et 9 mois. Quant au nombre de participations au total, l'étendue varic entre 6 fois et 72 fols, pour une moyenne de 22 fois.

Source : Racine ct al, (1997).

\section{Le plan d'analyse des données}

Trois des six types d'analyse de contenu développés par Aktouf (1987) se sont avérés appropriés pour cette recherche, c'est-à-dire l'analyse d'exploration de contenu (cerner des connaissances dans un domaine peu exploré), l'analyse de contenu qualitative (présence de thèmes, d'idées et de concepts), ainsi que l'analyse directe par la prise en considération du contenu manifeste uniquement (sens littéral des termes). L’analyse a principalement été basée sur les étapes définies par L'Écuyer (1987). Dans un premier temps, toutes les entrevues ont été transcrites mot à mot avec la précaution de préserver le style linguistique des interlocutrices et des interlocuteurs et de ne pas changer le sens de leurs propos. Par la suite, chacune des entrevues a été lue librement afin d'en dégager le sens global ainsi que les idées maîtresses, ce qui constitue en quelque sorte une 
préanalyse du matériel. Le choix des thèmes de la grille d'analyse a été à la fois basé sur les thèmes du guide d'entrevue et sur la première lecture des entrevues, permettant de développer des sous-catégories (catégorisation mixte). Par la suite, nous avons procédé à la réduction des données en éliminant les répétitions et les digressions anecdotiques. Chaque entrevue a par la suite été codifiée selon les thèmes de la grille d'analyse, tout en demeurant sensible à l'émergence de thèmes nouveaux. La fiabilité de la grille de codes a été testée par un accord inter-juges (Miles \& Huberman, 1994). Au premier essai, les trois juges indépendantes sont parvenues à un accord de $76 \%$; les thèmes de la grille ont été modifiés en conséquence. Au second essai, le pourcentage d'accords s'est élevé à $90 \%$, Le degré de cohérence interne, c'est-à-dire le degré d'accord à la suite d'une recodification d'extraits d'entrevues par la même personne (Miles \& Huberman, 1994),

La recherche qualitative commande une analyse différente que la simple quantification. Ainsi, pour l'ensemble de la recherche, les résultats qui touchent une majorité de participantes et participants (50\% et plus) seront nommés par le vocable tendance centrale ou majoritaire, alors que les points de vue moins fréquemment mentionnés seront nommés tendance marginale ou minoritaire (Deslauriers, 1991). Il est donc possible de voir apparaitre une ou plusieurs tendances centrales (réponses non mutuellement exclusives) ainsi que plusieurs tendances marginales. Notons que les tendances minoritaires comprennent de grandes variations quant à leur présence ou à leur intensité.

\section{RÉSULTATS}

\section{Les formes plurielles des cuisines collectives}

Le tableau 5 montre les principales caractéristiques des cuisines collectives qui ont collaboré à 1'étude; elles ont une origine, des caractéristiques et un fonctionnement pluriels. Parmi les 13 cuisines ayant participé au projet, on peut noter que 10 d'entre elles sont gérées par un organisme communautaire et que plus de la moitié peuvent compter sur le concours d'une personne salariée, engagée de façon permanente dans l'organisme, pour structurer leurs actions.

\section{Les motivations à participer à une cuisine collective}

Tel qu'illustré au tableau 6, une des principales motivations à participer à une cuisine collective est d'ordre économique. On $s^{t} y$ engage donc pour l'apport financier qu'elles procurent grâce à la confection de repas économiques et aux dépannages (colis de nourriture) qui les accompagnent souvent. Ensuite, la cuisine représente, pour beaucoup de personnes rencontrées, le moyen idéal de développer de nouvelles habiletés culinaires, de mieux se débrouiller du point de vue alimentaire et ce, dans un cadre non contraignant. Finalement, c'est pour briser leur isolement social que nombre de participantes et de participants se sont engagés dans ce type d'activité collective. Ces motivations sont celles ayant affiché une tendance centrale, tel qu'illustré par l'extrait suivant.

J'ai commencé pour des raisons économiques au départ. La motivation première, c'était ça. Mais avec le temps, c'est deveru autre chose. Il y a le côté 
REVUE CANADIENNE DE SANTÉ MENTALE COMMUNAUTAIRE

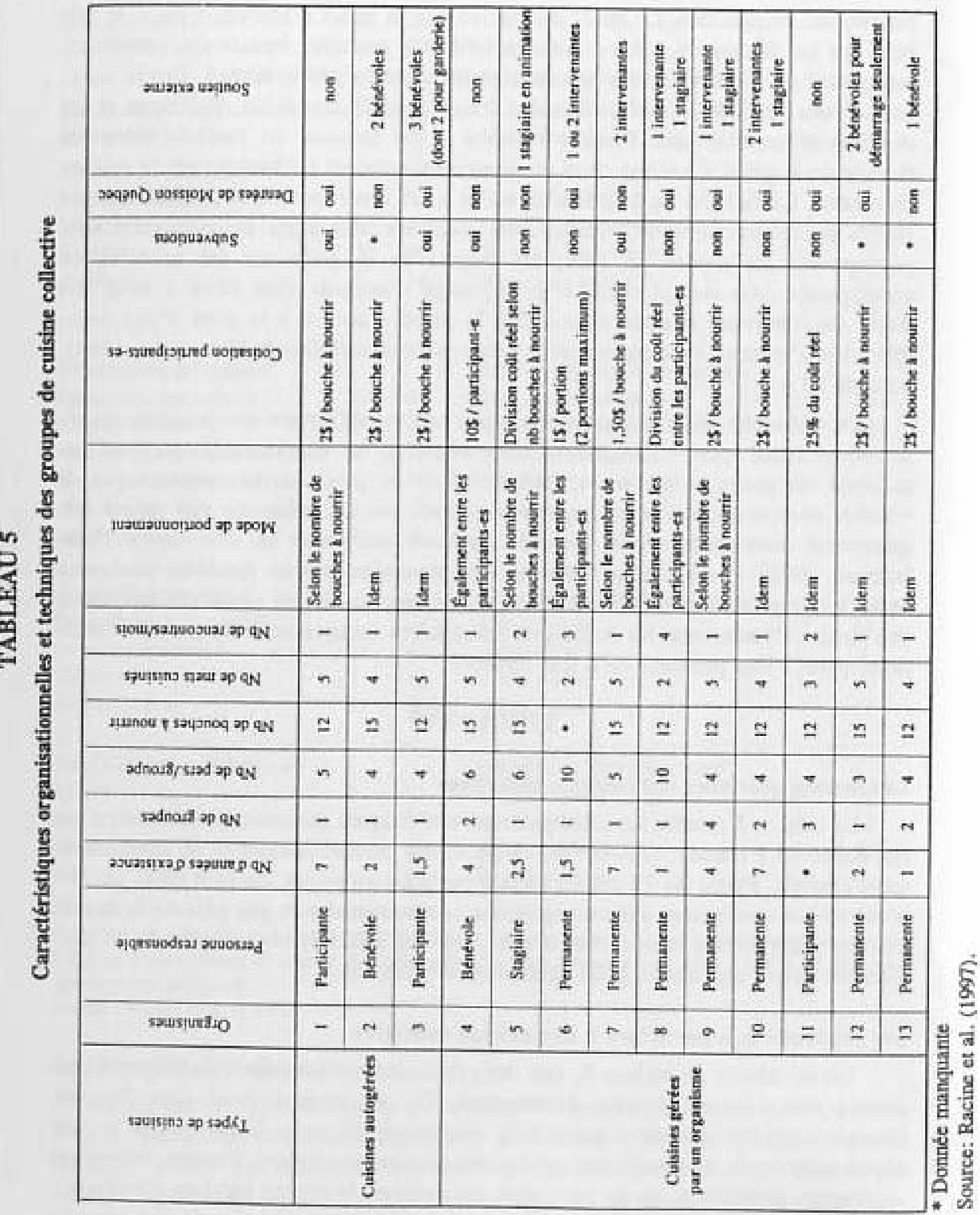




\section{LES CUISINES COLLECTIVES}

économique, mais il y a tout le côté *relations d'amitiés. C'est un beau climat, on est bien là-dedans (Entrevue \#25).

\section{La situation préalable des participantes et des participants}

La situation des personnes avant qu'elles ne s'engagent dans une cuisine collective est un facteur important à considérer car elle permet une analyse plus juste des retombées de la participation, de façon à cerner leur évolution dans le temps. Nous avons plus particulièrement focalisé sur l'état de santé mentale des personnes avant qu'elles ne prennent la décision de s'engager dans des activités de cuisine collective. Elles ont principalement mentionné l'isolement social comme étant un des éléments les plus perturbateurs de leur vie.

\section{TABLEAU 6}

Motivations à participer à une cuisine collective

\begin{tabular}{|c|c|}
\hline Motivations & Illustrations \\
\hline $\begin{array}{l}\text { Économiques } \\
\text { (majoritaire) }\end{array}$ & $\begin{array}{l}\text { - Combler les manques financiers* } \\
\text { - S'assurer une meilleure sécurité alimentaire }\end{array}$ \\
\hline $\begin{array}{l}\text { Nutritionnelles et } \\
\text { alimentaires } \\
\text { (majoritaire) }\end{array}$ & $\begin{array}{l}\text { - Apprendre à cuisiner ou à mieux se débrouiller en cuisine* } \\
\text { - Développer de nouvelles habiletés culinaires* } \\
\text { - Connaître de nouveaux mets et aliments (nouvelles idées)* } \\
\text { - Obtenir une meilleure variété alimentaire } \\
\text { - Développer des connaissances en nutrition } \\
\text { - Rapporter des mets cuisinés afin de prendre une pause } \\
\text { - Cuisiner en groupe et se motiver à cuisiner chez soi }\end{array}$ \\
\hline $\begin{array}{l}\text { Psychosociales } \\
\text { (majoritaire) }\end{array}$ & $\begin{array}{l}\text { - Briser l'isolement* } \\
\text { - Améliorer l'état de son moral en général (bien-être } \\
\text { psychologique) }\end{array}$ \\
\hline $\begin{array}{l}\text { Sociales et collectives } \\
\text { (minoritaire) }\end{array}$ & $\begin{array}{l}\text { - Entretenir des relations sociales à l'extérieur du réseau } \\
\text { habituei* } \\
\text { - Connaitre de nouvelles personnes dans le quartier } \\
\text { - Expérimenter le travail en équipe } \\
\text { - Développer des compétences sociales } \\
\text { - Partager une vie de groupe }\end{array}$ \\
\hline $\begin{array}{l}\text { Réseau de soutien } \\
\text { (minoritaire) }\end{array}$ & $\begin{array}{l}\text { - Développer un réseau d'entraide, de partage de services et } \\
\text { de soutien moral }\end{array}$ \\
\hline $\begin{array}{l}\text { Autres motivations } \\
\text { (minoritaire) }\end{array}$ & $\begin{array}{l}\text { - Développer son autonomie et son indépendance" } \\
\text { - Vivre une nouvelle expérience } \\
\text { - Exprimer sa créativité } \\
\text { - Partager ses connaissances et habiletés } \\
\text { - Se rendre utile à d'autres personnes }\end{array}$ \\
\hline
\end{tabular}

- Motivations qui ont été les plus fréquemment mentionnćes dans la catégorie correspondante. Source: Racine et al. (1997). 
J'étais toujours toute seule à la maison, les enfants étaient à l'école, mais j'avais encore ma dernière à la maison qui était toute petite. J'avais hâte de sortir, car j'étais toute seule avec la petite dans la maison. J'avais une auto, mais ça prend du gaz si tu veux sortir. Et j'allais à l'hôpital aussi parce que ma dernière était malade. C'était le striet minimum. Je me sentais seule, J'avais vraiment besoin de sortir de mon monde. Les amis perdus de vue, plus de conjoint .... (Entrevue \#19).

Mais l'isolement se vit à divers degrés. Pour certaines personnes, il s'agissait de l'absence totale de réseau de soutien; pour d'autres, c'était davantage l'incapacité đu réseau existant à répondre aux attentes; et pour quelques-unes qui avaient déjà accès à un réseau de qualité, il y avait des lacunes au plan de la quantité et de la variété. Plusieurs des personnes rencontrées n'avaient pas de famille près d'elles, pas plus qu'un réseau social.

Un manque de confiance et une faible estime personnelle représentent d'autres éléments caractéristiques de l'état de santé mentale des participantes et des participants avant qu'ils ne s'engagent dans les cuisines collectives. Bien que la tendance ne soit pas aussi forte que pour l'isolement, plusieurs ont mentionné que leurs conditions de vie difficiles avaient engendré une perte de reconnaissance et d'appréciation de leurs capacités. Certaines ont également mentionné l'impact négatif des préjugés de la société en général (et également des proches) envers les personnes assistées sociales sur ce plan. Le manque de courage et de motivation et la peur de ne pas être accepté des autres sont d'autres éléments qui ont été soulignés à ce chapitre. À cela s'ajoutent une situation financière précaire d'où découle un stress important, de même que l'obligation de développer de multiples stratégies de débrouillardise et de survie (privations) pour arriver à vivre décemment.

Mon estime de moì n'était pas forte, ma confiance non plus. Quand j'ai commencé ma première cuisine, je ne parlais pas. Je laissais les autres expliquer. Je m'impliquais pour faire à manger, mais je ne levais pas le ton plus qu'une autre. Si quelqu'un me posait une question, je répondais, mais pas plus que ça. I ... ] Jétais dépriméc, épuiséc. Pour le dire honnétement, quand je suis arrivée ici, $\mathrm{j}$ 'avais la tête entre les deux jambes (Entrevue \#2).

Afin d'analyser le plus justement possible l'apport des cuisines collectives, nous avons demandé aux répondantes et aux répondants de nous parler de l'utilisation d'autres formes d'aide ainsi que de leur engagement social dans leur communauté. Il est ressorti que la majorité des participantes et participants utilisaient d'autres ressources, activités ou services de différentes natures (psychosociales, alimentaires, académiques, ete.), soit dans le même organisme que la cuisine collective, soit ailleurs dans leur quartier. De plus, environ le quart étaient engagés dans leur milieu à titre de membre d'un conseil d'administration ou d'un comité de travail.

\section{Les effets de la participation aux cuisines collectives}

Les effets des cuisines collectives sur les personnes qui y participent sont de divers ordres et de différents types. Ainsi, ils se distinguent non seulement quant à leur nature (effets psychosociaux, économiques, etc.), mais également quant à leur moment d'apparition dans le processus de participation et à leur lien direct ou pas 
avec les activités de cuisine collective. Conséquemment, les retombées perçues peuvent découler directement de la participation aux cuisines ou être le produit d'autres effets.

Comme le montre le tableau 7 , les effets d'ordre psychosocial, économique, nutritionnel et alimentaire, et ceux en lien avec la création ou la consolidation d'un réseau de soutien tels que perçus par les répondantes et les répondants, sont de tendance majoritaire. Plus précisément, la confiance, l'estime personnelle, le bris de l'isolement par le biais de liens affectifs significatifs, l'apport financier, la sécurité alimentaire, les habiletés culinaires, les nouveautés alimentaires, le développement de liens d'amitié. l'entraide et le soutien moral représentent les effets les plus souvent mentionnés par les participantes et les participants de l'étude. L'extrait d'entrevue suivant montre l'importance des retombées autres qu'alimentaires.

Ce n'est pas juste de la bouffe. Si c'était juste ça, je pense qu'on ne serait pas resté. Je ne sais pas mais, c'est tout le reste. C'est vraiment une entraide à la grandeur (Entrevue \#5).

Évidemment, tous ces effets sont solidement imbriqués les uns dans les autres et s'influencent mutuellement. Il s'agit d'un tout quasi indissociable dans lequel se chevauchent et se succèdent différents apports qui ont un impact réel sur les conditions de vie en général des participantes et des participants. Par exemple, par la sécurité alimentaire qu'elles procurent, les cuisines collectives permettent une diminution significative du stress et de l'anxiété, ce qui a d'autres effets sur le vécu des membres, notamment sur leur relations familiales et sociales. Indubitablement, le cheminement de tous ces effets culmine vers un point central, soit l'amélioration
du bien-être psychologique.

Ca revient toujours à la confiance et à l'estime de soi, l'entraide, le fait de se prendre en main, de partager, c'est vraiment global. Je ne pourrais pas seulement mettre le doigt sur une chose, parce que pour moi, ça a couvert le tout, le bien-être en gênéral (Entrevue \$2).

\section{Les facteurs d'influence des effets de la participation}

Concernant les mécanismes par lesquels les participantes et les participants parviennent à des transformations sur le plan de leurs conditions de vie et à conserver une bonne santé mentale, trois types de facteurs ont émergé de l'analyse: premièrement, la dynamique interne, soit les facteurs reliés aux modalités de fonctionnement et à l'organisation des cuisines collectives; ensuite, les facteurs personnels, soit les éléments relevant de la situation de vie des participantes et des participants; et finalement, l'utilisation d'autres services et l'engagement social des personnes ailleurs que dans l'activité de cuisine comme telle.

La dynamique interne. Les éléments dynamiques qui sont reliés au fonctionnement peuvent être gênéraux ou s'appliquer plus spécifiquement à certains effets. Par exemple, la stabilité du groupe a peu d'influence directe sur l'augmentation de l'estime personnelle, mais en a beaucoup sur la consolidation d'un réseau de soutien et sur le développement de relations de confiance. Trois catégories de facteurs liés à la dynamique interne sont apparus: ceux qui relèvent de l'action concrète des personnes, ceux qui relèvent directement du fonctionnement de la cuisine, et ceux qui s'arriment à l'approche des intervenantes. 


\section{TABLEAU 7}

Tendances et caractéristiques des effets de la participation

\begin{tabular}{|c|c|c|}
\hline Effets de la participation & Tendance & $\begin{array}{c}\text { Moment } \\
\text { d'apparition }\end{array}$ \\
\hline
\end{tabular}

$\begin{array}{llllll}\text { Maj. Min. CT } & \text { MT } & \text { LT } & \text { D } & \text { I }\end{array}$

Psychosociaux

- Confiance en soi

- Estime personnelle

- Bris de l'isolement (liens affectifs)

- Connaissance de soi

- Soupape (diminution de la pression)

- Diminution du stress et de l'anxiété

- Dépassement des craintes et difficultés personnelles

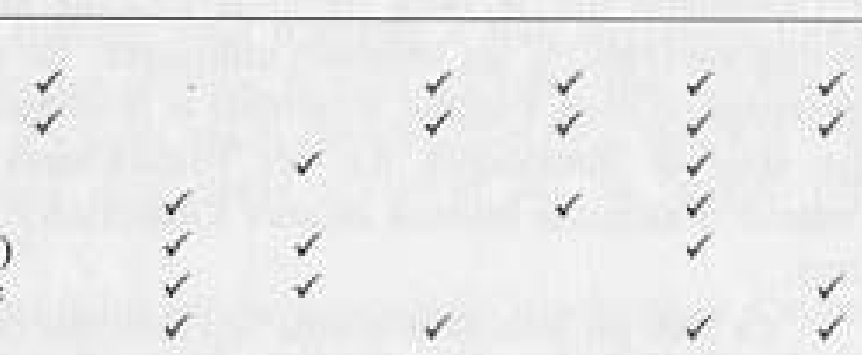

\section{Économiques}

- Apport économique (budget)

- Sécurité alimentaire

- Calcul du budget

- Habitudes d'achat et de consommation

\section{Nutritionnels et alimentaires}

- Habiletés culinaires

- Nouveautés alimentaires

- Connaissances en nutrition

\section{Reseau de soutien}

- Développement de liens d'amitié

- Entraide

- Soutien moral

- Connaissance des ressources du

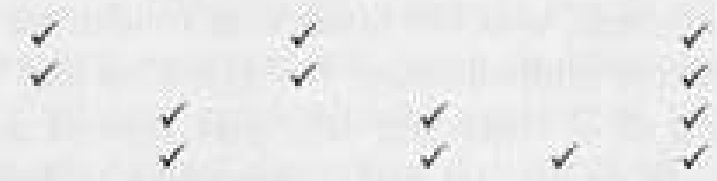
milieu

\section{Sociaux et collectifs}

- Sociabilité

- Intégration sociale

- Habiletés sociales, communautaires et collectives

\section{Autres effets}

- Organisation de la vie quotidienne

- Meilleures relations interpersonnelles

- Stimulation

- Effets récréatif5

- Acceptation de sa situation

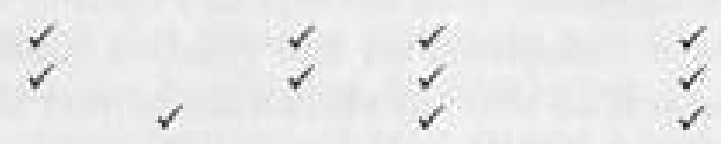

Note: Le moment d'apparition ainsi que lo lien de conséquence ne sont pas mutuellement exclusifs.

Légende: Tendance

Moment d'spparition

$\mathrm{Maj}=$ Majoritaire

Min. = Minoritaire

$\mathrm{CT}=$ Court terme

$\mathrm{MT}=$ Moyen terme

$\mathrm{LT}=$ Long terme.
Lien de conséquence $\mathrm{D}=$ Direct

$\mathrm{I}=$ Indirect

Source: Racine et al. (1997). 


\section{LES CUISINES COLLECTIVES}

Ces facteurs de dynamique interne sont tous reliés les uns aux autres et ils se conditionnent mutuellement. Si plusieurs effets dépendent de lambiance de la cuisine et de la qualité des relations interpersonnelles, ces dernières sont à leur tour conditionnées par d'autres facteurs tels la constitution du groupe ou l'approche des intervenantes (ouverture, souplesse, etc.). Il s'agit donc d'un processus dynamique où tous les éléments sont en constante interaction tel que le montre le tableau 8.

Selon les participantes et les participants, discuter et échanger sur sa situation personnelle est une chose importante, agir concrètement sur celle-ci en est une autre et c'est ce que les cuisines collectives permettent. Elles renferment pour les personnes qui y adhèrent la possibilité de mettre à profit leur potentiel et leurs capacités de façon tangible. Cela se traduit par l'équation expérimentation/réussite. En fait, c'est le processus de résolution de problème qui est à l'auvre ici.

petite réussite $\rightarrow$ capacités et confiance accrues $\rightarrow$ chances de réussite plus grandes

Les personnes se prouvent graduellement à elles-mêmes qu'elles ont le potentiel pour accomplir ce qu'elles désirent et reprennent graduellement du contrôle sur leur vie comme le montre l'extrait suivant.

Quand on fait une recette et qu'on la réussit, on est contente de notre coup.

C'est ça qui aide à développer notre confiance aussi, quand on fait quelque

\section{TABLEAU 8}

Facteurs de dynamique interne

$\begin{array}{cl}\begin{array}{c}\text { L'action concrète à travers } \\ \text { la participation }\end{array} & \text { - Sentiment d'utilité } \\ & \text { retirés } \\ \text { - Autonomie de gestion du groupe } \\ \text { - Haut degré de responsabilité }\end{array}$

- Qualité des relations interpersonnelles, ambiance et processus d'interaction*

- Configuration

Le fonctionnement de groupe

- Sentiment d'appartenance fort

- Base volontaire et engagement réel

- Stabilité

- Petit nombre de participantes et participants (intimité)

- Rotation et équité des tăches

- Nombre d'beures de participation et fréquence des rencontres relativement élevées

\section{L'approche des intervenantes}

- Principes reliés au démarrage des groupes

- Capacité d'adaptation et souplesse

- Consultation des membres (gestion participative)

\footnotetext{
- On notera également que certains des éléments soulevés représentent à la fois des effets de la participation et des facteurs d'émergence d'aures effets. Ils jouent ainsi un double rôle dans la dynamique des cuisines collectives. Par exemple, le soutien moral obtemu par les autres participantes et participants constitue un effet de la participation aux euisines, mais il représente également un facteur d'émergence de la confiance et de l'estime personnelle.

Source: Racine et al, (1997).
} 
chose et que ça marche. On se dit que si on a été capable de le faire une fois, on est capable de continuer et de réussir tout ce qu'on veut (Entrevue \#10).

Le sentiment d'utilité est un des éléments ayant ressorti le plus fortement en ce qui a trait à l'apparition des effets de nature psychosociale. Ce besoin de se sentir utile et apprécié, que les répondantes et les répondants ont fréquemment mentionné, est brimé par la situation de pauvreté qu'ils vivent, principalement les préjugés qui touchent les personnes prestataires de la sécurité du revenu. En revanche, les cuisines collectives offrent un milieu plus que propice à cet accomplissement personnel et collectif. Les personnes y évoluent dans un cadre de réciprocité et prennent graduellement conscience de leur capacité d'aide et d'entraide.

II y a des gens qui m'appellent et qui me demandent des conseils, qui m'appellent pour qu'on se voit et qu'on discute. Je regarde aussi l'utilité que j'ai au niveau de l'organisme pour les transports et tout ça. Tu sens que tu as une utilité, une valeur à quelque part .... (Entrevue \#18).

L'autonomie de gestion du groupe et le degré élevé de responsabilité des membres sont deux autres éléments ressortant très fortement quant à l'augmentation de la confiance et de l'estime personnelle. En fait, c'est tout le processus de gestion démocratique et participative qui est en cause. Les personnes expérimentent concrètement un pouvoir décisionnel, un des maillons du processus d'appropriation du pouvoir d'action selon Ninacs (1995).

Ça te rend autonome. C'est toi qui es responsable de différentes choses. Ça t'apprend à te débrouiller, à avoir des contacts, etc. Les cours [croissance personnelle] que j'ai pris là-bas aussi m'ont aidée. Mais ce n'était pas nous autres qui avaient la charge là-bas, ce n'est pas pareil. Être responsable et être assis pour écouter, ce n'est pas la méme chose (Entrevue \#14).

II convient de noter que le degré de responsabilité des participantes et des participants est tributaire des got̂ts et des capacités de chaque personne et que les dépasser peut constituer plus un danger qu'un avantage.

Je ne serais pas capable de gérer un groupe par exemple, je ne me sens pas assez... Il y a des choses que je serais capable de faire; je serais capable d'aider un autre groupe à partir, mais du point de vue des budgets et des finances, comment acheter et tout ça, je suis moins habituée. C'est sûr qu'arriver dans un nouveau groupe, dire que ça fonctionne de telle façon, je serais capable de le faire. Mais de là à gérer le groupe au complet, c’est une autre affaire, c'est une autre paire de manches. Je n'ai jamais voulu être etiboss» et ça ne m'intéresse pas. Je n'aime pas ça (Entrevue \#15).

En effet, il ne faut pas négliger l'importance du soutien que toutes les personnes ont affirmé recevoir dans l'accomplissement de leurs tâches et à travers leurs responsabilités. S'il est bénéfique, tant pour l'organisation que pour les personnes, que des responsabilités accrues soient assumées par les membres, cela doit impérativement s'accompagner d'un soutien et se réaliser graduellement. Si certaines personnes rencontrées ont eu la possibilité d'assumer des responsabilités de plus en plus grandes, c'est parce qu'elles ont eu la chance de bien s'intégrer au groupe à prime abord et de connaitre et comprendre assez bien le processus pour $s^{\prime} y$ sentir à l'aise, et parce qu'elles ont eu le temps de se familiariser avec l'ensemble de la démarche et de ses rouages. 
L'approche des intervenantes qui offrent un soutien aux cuisines collectives influence grandement ces deux derniers éléments (degré de responsabilité et autonomie du groupe). En outre, l'orientation a partir de laquelle les groupes ont démarré détermine leur fonctionnement ultérieur et l'esprit dans lequel les personnes s'engagent dans la cuisine collective. Un groupe basé sur l'entraide, la solidarité, la réciprocité, la gestion participative, l'acceptation mutuelle et la tolérance est plus prometteur au chapitre du développement personnel et collectif qu’un groupe qui ne démarre pas sur de telles bases.

\begin{abstract}
Tout au début, je n'ai pas beaucoup aimé ça, parce que la personne en charge me disait: Fais ci! Fais ça! Pourtant elle m'avait dit de prendre une recette et de la faire. J'ai pris la recette et elle me disait encore quoi faire, Je me demandais si ça allait marcher. Mais après, la deuxième fois, c'était une stagiaire et ça a bien été. La stagiaire fait la cuisine avec nous et elle nous demande comment on fait, elle nous demande notre opinion (Entrevue \#9).
\end{abstract}

Tous les éléments se rapportant à des caractéristiques de fonctionnement des cuisines collectives telles que la configuration et le nombre de membres par groupe, leur stabilité, la fréquence des rencontres, etc. représentent des éléments qui nécessitent une analyse plus détaillée puisque plusieurs nuances s'imposent. Par exemple, concernant la configuration du groupe, une certaine homogénéité semble bénéfique dans l'émergence de plusieurs des effets de nature psychosociale et dans l'établissement d'un réseau de soutien mutuel. Toutefois, un groupe hétérogène apparaît plus prometteur pour une multiplicité d'apprentissages étant donné les expériences diverses des personnes. Réunir des personnes ayant les mémes difficultés culinaires, par exemple, n'est pas souhaitable dans le processus que visent les cuisines collectives, soit le partage de connaissances entre les personnes.

Les facteurs personnels. Les éléments de nature personnelle, c'est-à-dire les motivations et les attentes de départ et la situation de vie des personnes avant leur participation aux cuisines collectives, ont une influence sur les retombées possibles ainsi que sur les variations d'intensité d'une personne à l'autre. Lorsqu'une personne choisit de faire partie d'une cuisine collective pour apprendre à cuisiner, c'est ce qu'elle va en retirer. En dépit de ces motivations préalables, les membres ont retiré davantage de leur expérience que ce qu'ils avaient prévu au départ. À l'inverse, quelques déceptions ont été notées.

Je me disais qu'entre nous, on allait se défouler. C'est partager sa pauvreté, partager sa difficulté quand on est humilié ou découragé. [ . . . ] Après avoir vu le vidéo des cuisines collectives à Montréal, comment ça se passait, sachant la difficulté đu monde à se nourrir de plus en plus, je m'attendais à autre chose, [ . . ] J'ai trouvé qu'il y avait un vide, un manque (Entrevue \#20).

La même analyse s'applique en ce qui concerne la situation de vie des participantes et des participants avant qu'ils ne s'engagent dans une cuisine collective. En effet, lorsque l'estime personnelle d'une personne était déja excellente au départ, sa participation aux cuisines collectives n'a pas influencé cet élément. À l'inverse, une participante ébranlée dans sa confiance perçoit des retombées sur ce plan en premier lieu.

L'utilisation d'autres services et les engagements sociaux. Concernant 1'influence d'autres activités et services utilisés par les participantes et les participants ou de leur engagement social (bénévolat principalement) sur les effets perçus, dans 
presque tous les cas, les répondantes et les répondants ont affirmé que c'est de l'ensemble des activités auxquelles ils participent que proviennent les changements observés, bien que certains à caractère nutritionnel, alimentaire et économique ne relèvent que de la cuisine collective. Bien qu'il ne soit pas possible de mesurer cette influence, les personnes rencontrées ont soulevé la prépondérance des cuisines collectives dans les effets perçus par rapport aux autres activités ou à leur engagement social. Cette hégémonie s'explique par quelques éléments qui caractérisent les cuisines collectives et qui ne se retrouvent pas ou peu dans d'autres activités communautaires, soit la possibilité de mettre concrètement en application des apprentissages et des prises de conscience personnelle, la durée de l'activité (une journée complète en général), l'ambiance particulière d'échange et de partage. le peu de formalisation dans le fonctionnement, etc.

\begin{abstract}
Mais toutes les autres choses sont arrivées après les cuisines collectives, Parce que les cuisines, j'ai commencé par là, ça a été mon départ, ma base. Je pense que si je ne m'étais pas embarquée dans les cuisines collectives, je ne me serais pas laissée aller vers les autres, parce que mon approche, je l'ai apprise dans les cuisines où il fallait que je travaille avec trois autres personnes. II fallait que je m'adapte là-dedans, apprendre à donner des feedback sur comment je me sentais et ça, je l'ai fait dans les cuisines, Les cuisines, ça a cassé la glace (Entrevue \#2).
\end{abstract}

Ce qui a été encore plus probant dans le contenu des entrevues, à l'instar des résultats de la recherche de Fréchette et Favreau (1997), c'est qu'à toutes les fois où les cuisines collectives se retrouvent dans un organisme où les personnes ont la possibilité de recevoir d'autres services ou de participer à d'autres activités, les effets sont généralement attribués par les rếpondantes et les répondants non pas à des services en particulier, mais plutôt à l'organisme en général, à l'approche et la philosophie d'action des intervenantes et des intervenants qui y évoluent. Noraz (1996) abonde dans le même sens en ce qui a trait à l'existence et au dynamisme d'autres organismes dans un même milieu, c'est-à-dire la vivacité du mouvement communautaire dans un quartier.

\title{
DISCUSSION
}

En examinant les résultats de la présente recherche quant aux retombées des cuisines collectives en lien avec les déterminants de la santé mentale (tableau 1), on constate qu'elles ont un impact sur six des neuf catégories proposées. Elles agissent principalement sur les valeurs sociales et culturelles par les grands principes de partage, d'échange, d'autonomie et de réciprocité qu'elles mettent en place, sur les ressources psychologiques personnelles par les habiletés acquises et l'augmentation de confiance et de l'estime personnelle et sur les relations de soutien en donnant accès à des sources variées et de qualité.

Les cuisines collectives agissent également, avec moins d'intensité toutefois, sur les conditions économiques. Certes, elles permettent des économies substantielles pour les ménages, mais ne changent pas leur revenu de base, ni ne leur donnent accès à un emploi satisfaisant. Il serait d'ailleurs intéressant d'explorer cette question de l'impact économique des cuisines collectives dans un quartier ou une région en tenant compte des dépenses des ménages dans d'autres secteurs d'activité économique grâce aux économies réalisées. Quant à l'apport des cuisines collectives sur les habitudes de vie et sur la quantité et la qualité des services, il est 
moins direct mais tout aussi important. En effet, les changements dans les habitudes alimentaires (consommation accrue de légumes par exemple) des membres des cuisines collectives et de leur famille sont fréquents, de même qu'une augmentation des ressources disponibles par la connaissance de celles-ci et les échanges de service entre les participantes et participants. Les résultats de notre recherche ne nous permettent pas de savoir si les cuisines collectives agissent sur l'environnement physique et politique ou sur d'autres éléments des conditions économiques, mais elles ont le potentiel pour le faire. Actuellement, seul le Regroupement des cuisines collectives du Québec (RCCQ) et quelques groupes plus politisés travaillent dans le développement économique communautaire et dans la défense de droits afin d'améliorer les conditions de vie des communautés touchées par la pauvreté. Ce chemin est déjà amorcé suite à la recherche de Fournier et al. (1998) où un outil d'éducation populaire a été créé permettant aux diverses cuisines collectives de réfléchir et d'agir sur ces situations. Toutes ces potentialités des cuisines collectives sont conditionnelles à des facteurs tels que I'orientation, la coordination et les valeurs de base qui sont déterminants dans la capacité des cuisines à devenir des stratégies de promotion de la santé mentale et aussi de changement social.

La dynamique en auvre dans les cuisines collectives relève clairement d'un processus d'aide mutuelle entre les participantes et participants et c'est ce qui fait la force de cette pratique. Elle a la capacité de réunir des personnes qui se sentent adans le même bateau et de leur permettre de vivre un processus d'échange qui leur ressemble, c'est-à-dire souple et spontané. Il ne s'agit pas d'un groupe formel d'entraide ou de discussion où chaque personne risque de se présenter avec des attentes plus précises sur le plan du soutien ou de la résolution de problèmes personnels. Et c'est un peu la magie des cuisines collectives que de permettre cette dynamique d'aide mutuelle tout en ne la formalisant pas, d'offrir à des femmes et à des hommes un lieu de partage ouvert et rempli de potentiels. Qu'on veuille créer des contacts, apprendre de nouvelles recettes ou développer sa confiance, les cuisines collectives, par le processus d'aide mutuelle qui les caractérisent, permettent tout cela.

Cette dynamique est toutefois conditionnée par l'action des intervenantes qui soutiennent les cuisines collectives (professionnelles, bénévoles ou bénévoles-participantes) et par les bases sur lesquelles le groupe a été mis sur pied. La dynamique d'aide mutuelle et l'autonomie que le groupe pourra atteindre peuvent être encouragées ou au contraire entravées par l'intervention des personnes qui gravitent autour des participantes et des participants. Par exemple, une responsable qui part avec le postulat que les personnes vivant en situation de pauvreté ne savent pas s'organiser et qu'il faut tout leur apprendre aura un impact important sur la dynamique du groupe. Si les groupes visent l'autonomie et la prise en charge, l'intervention devrait aller dans le sens du développement de l'aide mutuelle et done du partage d'expériences et de connaissances entre les personnes, plutôt que dans le sens d'une relation de type professeur-élève.

$\mathrm{Si}$ on se réferre au processus d'appropriation du pouvoir d'action tel que défini par Ninacs (1995), du point de vue individuel, une personne passe d'une conscience personnelle à une conscience collective. Tous les aspects réunis (conscience critique, estime de soi, compétences techniques et participation) permettent 
de passer concrètement à l'action pour changer des situations et avoir un meilleur contrôle sur son environnement. Le développement de la conscience critique semble le maillon faible des cuisines collectives de la région de Québec. En effet, sur les groupes de cuisines qui ont participé à notre recherche, aucun ne travaillait spécifiquement cet aspect. Cela semble en partie dâ à la définition et à la façon d'implanter les cuisines collectives sur un territoire donné. Se référant aux recherches de Fréchette et Favreau (1997) et de Noraz (1996), on remarque que la définition de ce qu'est une cuisine collective diffère de celle employée dans notre recherche. Pour ces derniers, une cuisine collective peut être un organisme communautaire où évoluent des personnes participantes, employées, militantes et bénévoles, et où se pratiquent diverses activités autour de celles de cuisine collective proprement dites. À Québec, rares sont les organismes communautaires qui se sont développés autour des cuisines collectives. C'est plutôt le contraire qui s'est produit; les cuisines collectives se sont greffées aux services et aux activités d'organismes déjà en place. Une cuisine collective, dans ce cas-ci, représente le noyau ou la cellule composée des quelques personnes qui cuisinent ensemble des repas sains et économiques. On doit donc établir une distinction claire entre les activités de cuisine collective et tout ce qui peut se développer autour de celles-ci car les données recueillies, ainsi que les analyses que l'on peut en faire, vont différer substantiellement.

Les cuisines collectives peuvent être bien davantage qu'une forme d'aide alimentaire parmi d'autres. Leur potentiel d'intégration sociale et surtout de changement social reste encore à développer. Mais pour qu'elles permettent minimalement un développement personnel constructif (estime, confiance, connaissances, etc.), elles doivent mettre de l'avant des principes et des valeurs de solidarité, de responsabilité, de coopération et de respect. C'est lors du démarrage des groupes que de telles bases doivent être jetées, afin que les membres prennent en charge leur groupe et le mènent vers la destination qu'ils auront collectivement choisie.

Il existe d'ailleurs un danger de vouloir faire des cuisines collectives des pratiques institutionnelles normatives pour favoriser le développement personnel de clientèles particulières, c'est-à-dire de développer des cuisines où l'expertise, la créativité, la spontanéité et la culture des personnes auraient peu de place. Les cuisines collectives représentent d'abord et avant tout une expérience communautaire qui mise sur les forces de personnes plutôt que sur leurs difficultés et dont les caractéristiques de souplesse et d'adaptabilité sont à mettre de l'avant à la lumière de nos résultats. Le fait qu'elles ne soient pas les seules à produire les différents effets perçus montre en fait que, vidées de leur subtance et sorties de leur milieu. elles ne seraient pas les mêmes. Cela nous amène à la pertinence de l'intervention professionnelle (nutrition, organisation communautaire, etc.) dans les pratiques des cuisines collectives. Il s'agit d'un enjeu très délicat dans la mesure où il semble comporter autant d'avantages que d'inconvénients. Un groupe tout à fait autonome de participantes et de participants développe davantage son sentiment de responsabilité et ses capacités de gestion, mais sans une animation extérieure, il semble stagner dans son développement communautaire. Encore une fois, la question est de savoir ce qu'on veut faire des cuisines collectives et l'orientation qu'on souhaite leur voir prendre, car il y a bien plus que le développement personnel dans cette pratique. Perçue comme un palliatif au filet de sécurité sociale ou comme pratique 


\section{LES CUISINES COLLECTTVES}

de développement communautaire axée sur l'entraide, la solidarité et le changement social, l'intervention à choisir differera grandement. En somme, l'intervention professionnelle (animation planifiée) peut avoir sa pertinence dans la mesure où elle ne se substitue pas à l'action des participantes et des participants et qu'elle favorise la dynamique d'aide mutuelle.

\section{CONCLUSION}

Plusieurs interrogations demeurent suite à cette étude. Par exemple, la question de la permanence des effets après que les personnes aient quitté les cuisines demeure entière. L'impact des effets reliés à la création d'un réseau de soutien par les cuisines collectives et celui de l'effet de soupape que les cuisines collectives procurent aux participantes et aux participants (qui ont fréquemment mentionné que d'aller aux activités de cuisine collective leur permettait d'évacuer la pression accumulée pendant le mois) sont des éléments préoccupants dans la mesure où on peut se demander si les personnes redeviendront déprimées, épuisées et isolées lorsqu'elles auront quitté le groupe de cuisine.

Les effets à plus long terme des cuisines collectives comme activité concrète d'entraide (emploi, retour aux études, etc.) est également une question qui demeure en suspens. Il serait intéressant, dans une recherche subséquente, d'évaluer ces aspects auprès des personnes qui ont quitté les cuisines collectives depuis un certain temps pour cerner dans quelle mesure les habiletés acquises ont été sauvegardées ou consolidées avec le temps.

Les dépannages alimentaires (colis de nourriture) qui accompagnent fréquemment les activités de cuisine collective nous interrogent quant à leur impact sur les effets économiques, dans un premier temps, et sur l'autonomie des groupes et des participantes et participants, dans un deuxième temps. On peut se demander si à long terme ces dépannages ne risquent pas d'engendrer une forme de adépendancer, ce que les cuisines collectives rejettent dans leurs principes de base. Par contre, nous savons que les motivations des membres à s'engager dans des cuisines collectives sont étroitement liées à la possibilité de faire des économies et que sans les denrées de Moisson Québec ou les diverses subventions reçues, elles seraient beaucoup moins importantes. Cela nous ramène done à la nécessité de travailler à de réels changements sociaux (développement social durable) pour que la situation des personnes se transforme à la base en leur assurant de meilleurs revenus.

La durée de la participation aux cuisines collectives est un autre facteur qui nous interpelle, À notre avis, cela comporte d'autres dangers à long terme. Bien que les persomes participent concrètement aux cuisines et gèrent leur groupe, il nous semble qutune participation à long terme risque de freiner le processus d'autonomie et d'appropriation du pouvoir d'action. Étant donné que les membres, même après plusieurs années d'engagement au scin des cuisines, ne semblent pas enclins à quitter les groupes ou même à en créer de nouveaux de façon complètement autonome, on peut se demander si les cuisines collectives ne deviennent pas à long terme des substituts de participation sociale. Mais cela dépend évidemment de ce que l'on fait des cuisines collectives et des objectif's visés par les groupes. II n’est pas possible d'utiliser les termes de edépendances ou de eservice lorsqu'un groupe se gère lui-même et dépasse largement le simple dépannage alimentaire. 
D'ailleurs, on sait que certaines cuisines collectives se sont transformées en entreprises d'économie sociale et que d'autres poursuivent de plus en plus des objectifs sociopolitiques en faisant pression pour des changements substantiels et durables. La visée à long terme d'une cuisine collective devrait selon nous dépasser le développement personnel, bien que non négligeable, pour évoluer du côté des changements collectifs et sociaux. Beaucoup de débats sont encore à tenir sur cette pratique jeune et prometteuse.

Nous croyons que la dynamique interne des cuisines collectives permettant l'émergence de différents effets a un potentiel à produire le même type de retombées dans d'autres activités à caractère alimentaire. Les éléments composant ce processus dynamique doivent être adaptés à la réalité de chaque organisme afin d'améliorer les conditions de vie des personnes de multiples façons. Ceux-ci ne sauraient en aucun cas constituer une recette miracle dont l'amalgame des ingrédients suffirait à produire les mêmes effets. La réalité du terrain est complexe et nécessite une réflexion et une action adaptée à chaque milieu. Un organisme qui désirerait transformer sa pratique en se basant sur les facteurs d'émergence des effets que nous avons soulevés devrait, à notre avis, débuter son action par des réflexions et des débats avec toutes les personnes concernées, principalement celles qui sont directement touchées par une situation de pauvreté.

Selon les participantes et les participants rencontrés, les cuisines collectives représentent une forme d'entraide alimentaire intéressante et stimulante qui leur apporte de nombreux bénéfices, en plus de celui de s'assurer une meilleure sécurité alimentaire. Bien que les cuisines collectives agissent concrètement sur les conditions de vie des personnes qui s'y engagent et qu'elles leur apportent une plus grande autonomie sans affecter leur dignité, elles ne constituent pas une panacée face au problème de la pauvreté.

\section{ABSTRACT}

Collective kitchens represent a relatively new social and community practice in the province of Quebec. They provide support that goes far beyond the food they supply, affecting the participants' life conditions and personal development. This qualitative study aimed to evaluate the effects that collective kitchens have on participants. We carried out semistructured interviews with a stratified sample of 25 people between the ages of 20 and 60 , a majority of whom were single parents. These 20 women and 5 men were involved in 13 different groups for an average of 1 to 2 years. The results showed that the main reasons for participating in these groups were economic, nutritional, and psychosocial in nature. Prominent among the psychosocial reasons was a desire to overcome a fecling of isolation, which a majority of respondents perceived as one of the most disturbing features of their lives before they were involved in the collective kitchens. We identified 3 types of factors that influence the effects of the respondents' participation in collective kitchens: (a) factors telated to the internal dynamics of each collective kitchen (their modes of functioning and organizational structure), (b) factors related to the personal . situation of the participants and (c) factors related to the use of other services or to the participants' social involvement. The various effects differ not only in character (psychosocial, economic, nutritional, etc.) but also in the time they appeared in the participation process and in their direct or indirect links with participation in collective kitchens. It would be interesting in future research to 


\section{LES CUISINES COLLECTTVES}

evaluate whether the benefits of participation in collective kitchens identified in this study have a long-term impact on the lives of the people in these groups.

\section{RÉFÉRENCES}

Aktouf, O. (1987). Methodologie des sciences sociales et approche qualitative des organisations. Sillery, QC: Presses de l'Université du Québec.

Association des cuisines collectives et créatives de la région de Québec. (1999). Liste des cuisines collectives et créatives de la région de Québec. Document inédit.

Beaud, J.-P. (1990). Les techniques d'échantillonnage. Dans B. Gauthier (dir.), Recherche sociale: de la problématique à la collecte des données (pp. 175-200). Sillery, QC: Presses de 1'Université du Québec.

Beeman, J., Panet-Raymond, J., \& Rouffignat, J. (1997). Du dépannage alimentaire au développement communautaire: des pratiques altematives. Cahier de fiches. Montréal: Université de Montréal, École de service social.

Blanchet, L., Laurendeau, M.-C., Paul, D., \& Saucier, J.-F. (1993). La prévention et la promotion en santé mensale: préparer l'avenir. Boucherville, QC: Comité de la santé mentale du Québec \& Gaëtan Morin Éditeur.

Conseil national du bien-être social. (1998). Profil de la pauvreté 1996. Ottawa: Auteur.

Deslauriers, J.-P. (1991). Recherche qualitative: guide pratique. Montréal: McGraw-Hill.

Deslauriers, J.-P. (1998). Les cuisines collectives, c'est plus que la cuisine! Hull, QC: Université du Québec à Hull, Département de travail social, Groupe d'étude et de recherche en intervention sociale.

Fournier, D., Provost, M., \& Goudreault, N. (1998). Pauvrelé el aulonomie sociale: les cuisines collectives comme stratégie de solidarité (Rapport de recherche). Montréal: Relaisfemmes \& Regroupement des cuisines collectives du Québec.

Fréchette, L., \& Favreau, L. (1997), Les cuisines collectives: une intervention au carrefour de l'entraide et du développement local (Rapport de recherche). Hull, QC: Université du Québec à Hull, Groupe d'étude et de recherche en intervention sociale, pour le CLSC Vallée-de-la-Lièvre.

Kalina, L. (1993), Community kitchens, Kamloops Food Share (Rapport de recherche). Kamloops, BC: Kamloops Food Share.

L'Écuyer, R. (1987) L'analyse de contenu: notions et étapes. Dans J.-P. Deslauriers (dir.), Les méthodes de la recherche qualitative (pp. 49-65), Sillery, QC: Presses de l'Université đu Québec.

London Good Food Project. (1993). Collective kitchen: Participatony research (Rapport d'évaluation). London, ON: Auteur.

Mayer, R., \& Ouellet, F. (1991). Méthodologie de recherche pour les intervenants sociaux. Boucherville, QC: Gaétan Morin Éditeur.

Miles, M.B., \& Huberman, A.M. (1994). Qualitative data analysis (2nd ed.). Thousand Oaks, CA: Sage Publications.

Ministère de la Santé et des Services sociaux du Québec. (1990). La promotion de la santé mentale. Santé Sociëté, cahier 5, 10-11.

Mirowski, J., \& Ross, C.E. (1989). Social causes of psychological distress. New York: Aldine de Gruyter.

Moisson Québec. (1999). Statistiques de la faim en chiffres avril 1999. Québec: Auteur.

Montminy, J. (1991). Guide pour la mise en place des cuisines collectives. Québec: CLSC Laurentien.

Ninacs, W.A. (1995). Empowerment et service social: approches et enjeux. Service social, 44(1), 69-93. 
Noraz, C.-A. (1996). Processus et stratégies d'insertion sociale dans un projet communautaire: le cas de la Cuisine collective Hochelaga-Maisonneuve. Montréal: Université đu Québec à Montréal, Cahiers du CRISES, Collection thèses et mémoires.

Racine, S., Côté, É, Morrow, C., St-Onge, M., \& Tremblay, E. (1997). Santé mentale et pauvreté: les cuisines collectives, une altemative? (Rapport de recherche présenté à la Régie régionale de la Santé et des Services sociaux de Québec). Québec: Moisson Québec \& Centre de santé publique de Québec.

Regroupement des cuisines collectives du Québec. (1996). Guide de fonctionnement des cuisines collectives. Montréal: Auteur.

Renaud, C. (1993). Du phénomène de l'entraide au processus d'aide mutuelle en service social des groupes. Lise Tessier (dir. de la série), Cahiers du service social des groupes, $\mathrm{n}^{\circ}$ 6. Québec: Université Laval, École de service social.

Robichaud, J.-B., Guay, L., Colin, C., \& Pothier, M. (1994). Les liens entre ta paurvreté et la santé mentale: de l'exclusion à l'équité. Boucherville, QC: Comité de la santé mentale du Québec \& Gaëtan Morin Éditeur.

Santé Québec. (1989). Et la Santé, ça va? Rapport de l'enquête Santé-Québec 1987. Québec: Les publications du Québec.

Santé Québec. (1994). Et la Santé, fa va? Rapport de l'enquéle Santé-Québec 1992-93. Québec: Les publications du Québec.

Tremblay, E. (1995). Portrait des cuisines collectives de la région de Québec. Essai de maitrise, Université Laval, Québec. 